\title{
Optical imaging to trace near infrared fluorescent zinc oxide nanoparticles following oral exposure
}

This article was published in the following Dove Press journal:

International Journal of Nanomedicine

26 June 2012

Number of times this article has been viewed

\section{Chang-Moon Lee ${ }^{1-4, *}$ \\ Hwan-Jeong Jeong ${ }^{1-4, *}$ \\ Kuk-No Yun ${ }^{1-3}$ \\ Dong Wook Kim ${ }^{1-4}$ \\ Myung-Hee Sohn ${ }^{1-4}$ \\ Jong Kwon Lee \\ Jayoung Jeong ${ }^{5}$ \\ Seok Tae Lim ${ }^{1-4}$}

*These authors contributed equally to this work.

'Department of Nuclear Medicine;

${ }^{2}$ Cyclotron Research Center;

${ }^{3}$ Research Institute of Clinical

Medicine; ${ }^{4}$ Institute for Medical

Sciences, Chonbuk National

University Medical School and

Hospital, Jeonju, Jeollabuk-Do,

Republic of Korea; ${ }^{5}$ Toxicological

Research Division, National Institute

of Food and Drug Safety Evaluation,

Osong-Eup, Chungchungbuk-Do,

Republic of Korea
Correspondence: Seok Tae Lim Department of Nuclear Medicine, Chonbuk National University Medical School and Hospital, 634-18,

Geumam-2 Dong, Dukjin-Gu, Jeonju, Jeonbuk 56I-7I2, Republic of Korea

Tel +8263250 I I 72

Fax $+8263255 \quad$ II72

Email stlim@jbnu.ac.kr
Background: Understanding how nanomaterials are distributed in the body after exposure is important for assessing whether they are safe. In this study, we investigated the behavior and accumulation of nanoscaled and submicron-scaled zinc oxide $(\mathrm{ZnO})$ particles in the body using optical imaging following oral exposure.

Methods: To trace these nanoparticles in the body, $\mathrm{ZnO}$ nanoparticles were conjugated with a monoreactive hydroxysuccinimide ester of Cy5.5 (Cy5.5-NHS), and the conjugation-stabilizing effect of Cy5.5 on the nanoparticles was evaluated in simulated gastric fluid ( $\mathrm{pH} 1.2$ ) for 7 hours. To compare the distribution of Cy5.5-NHS and Cy5.5-conjugated ZnO nanoparticles, Cy5.5NHS $0.5 \mathrm{mg} / \mathrm{kg}$ and Cy5.5-conjugated $\mathrm{ZnO}$ nanoparticles $250 \mathrm{mg} / \mathrm{kg}$ were administered orally to healthy rats. We collected blood from the rats at predesignated time points for 7 hours after administration, and optical imaging studies were performed at 1,2, 3, 5, and 7 hours after dosing. To investigate the extent of nanoparticle accumulation in the organs and tissues, the mice were sacrificed at 23 hours after administration, and the organs were removed and imaged.

Results: Cy5.5-conjugated $\mathrm{ZnO}$ nanoparticles were stable in simulated gastric fluid for 7 hours. The signal intensity of Cy5.5-NHS in blood was highest 3 hours after oral administration, and Cy5.5-conjugated $\mathrm{ZnO}$ nanoparticles showed the highest signal intensity in blood 5-7 hours after administration. In vivo optical images indicated that Cy5.5-NHS showed optical signals in the lung, liver, and gastrointestinal tract after oral administration, whereas Cy5.5-conjugated $\mathrm{ZnO}$ nanoparticles were seen only in the gastrointestinal tract. Seven hours following administration, biodistribution studies demonstrated that Cy5.5-NHS accumulated in the lung and liver, and Cy5.5-conjugated $\mathrm{ZnO}$ nanoparticles resulted in a strong signal in the kidney and liver. Different-sized $\mathrm{ZnO}$ nanoparticles showed dissimilar patterns of biodistribution in ex vivo optical images.

Conclusion: $\mathrm{ZnO}$ nanoparticles are absorbed into the tissues following oral exposure and their behavior can be monitored and evaluated using optical imaging.

Keywords: zinc oxide nanoparticles, biodistribution, optical imaging, oral administration

\section{Introduction}

Molecular imaging, defined as characterization and measurement of biological and cellular processes in vitro and in vivo, is a noninvasive approach for monitoring the biodistribution and interactions of a molecule in vivo. ${ }^{1}$ Among the various molecular imaging modalities, noninvasive optical imaging enables tracking and monitoring of molecules entering the body with potentially higher sensitivity and specificity than other imaging modalities. ${ }^{2,3}$ The recent wide application of nanomaterials in various fields has led us to address the potential risks of these materials to human health. ${ }^{4}$ Molecular imaging techniques, including optical imaging, may increase our understanding of the 
behavior and accumulation of nanomaterials in the body following exposure via various routes.

Zinc oxide $(\mathrm{ZnO})$ nanoparticles have a wide variety of applications in industry, including agriculture, medicine, and cosmetics. ${ }^{5}$ With increased use of $\mathrm{ZnO}$ nanoparticles, exposure to these nanoparticles has been rising steadily, resulting in more attention being paid to their potential toxicity, including cytotoxic, genotoxic, and proinflammatory effects. ${ }^{6,7}$ Several studies have reported that $\mathrm{ZnO}$ nanoparticles at a high dose of $1-5 \mathrm{~g} / \mathrm{kg}$ can cause acute toxicity and apoptosis in murine liver cells in vivo. ${ }^{8,9}$ Although a toxic potential for $\mathrm{ZnO}$ nanoparticles has been suggested, our understanding of their actual toxicity is not yet adequate. Understanding the pharmacokinetics and clearance of $\mathrm{ZnO}$ nanoparticles from the body following exposure is essential when assessing their potential for toxicity. Movement and translocation of nanoparticles after absorption into the body can be monitored and traced noninvasively by tagging with a fluorescent dye or labeling with a radioisotope. ${ }^{10}$

In this study, we investigated the behavior and biodistribution of near infrared fluorescent dye-labeled nanoscaled and submicron-scaled $\mathrm{ZnO}$ nanoparticles following oral exposure in rats using an optical imaging system. To conjugate a near infrared fluorescent dye, $\mathrm{ZnO}$ nanoparticles were chemically modified and their conjugation stability in gastric conditions was evaluated.

\section{Materials and methods Materials}

$\mathrm{ZnO}$ nanoparticles (nanoscale $20 \mathrm{~nm}$ and submicron scale $100 \mathrm{~nm}$ ) were obtained from Sumitomo (Tokyo, Japan) and American Elements (Los Angeles, CA), respectively. Their size distribution, determined by dynamic light scattering, was $25.4 \pm 3.9 \mathrm{~nm}$ for the $20 \mathrm{~nm}$ size and $78.9 \pm 13.4 \mathrm{~nm}$ for the $100 \mathrm{~nm}$ size, respectively. Sodium citrate, HEPES buffer, 2.2' -(ethylenedioxy)bis-ethylamine (EBEA), and $\mathrm{N}$-(3dimethylaminopropyl)- $N$-ethylcarbodiimide hydrochloride were purchased from Sigma-Aldrich Chemical Company (St Louis, MO). A monoreactive hydroxysuccinimide ester of Cy5.5 (Cy5.5-NHS) was obtained from GE Healthcare (Uppsala, Sweden).

\section{Preparation of Cy5.5-conjugated $\mathrm{ZnO}$ nanoparticles}

Nanoscaled or submicron-scaled $\mathrm{ZnO}$ nanoparticles (10 mg) were suspended in $5 \mathrm{~mL}$ of HEPES buffer (0.02 M, pH 7.0) containing $5 \mathrm{mg}$ of sodium citrate. After sonication for $30 \mathrm{sec}-$ onds, the $\mathrm{ZnO}$ nanoparticles were collected by centrifugation at 10,000 rpm for 5 minutes and washed with HEPES buffer three times. To introduce amine groups to the Cy5.5 conjugate, citrate-ZnO nanoparticles were chemically modified with EDEA. The introduction of amine groups onto citrate- $\mathrm{ZnO}$ nanoparticles was characterized by Fourier transform infrared spectroscopy. The EDEA-modified citrate- $\mathrm{ZnO}$ nanoparticles were suspended into $5 \mathrm{~mL}$ of sodium borate buffer $(0.1 \mathrm{M}$, $\mathrm{pH}$ 8.5), and Cy5.5-NHS (0.1 mg) in dimethyl sulfoxide $(10 \mu \mathrm{L})$ was added under stirring. The solution was stirred for 6 hours at room temperature in the dark. The Cy5.5-conjugated $\mathrm{ZnO}$ nanoparticles were collected and washed by centrifugation. To investigate the separation of Cy5.5 from the $\mathrm{ZnO}$ nanoparticles when Cy5.5-conjugated $\mathrm{ZnO}$ nanoparticles were orally administered in the rat, a conjugation stability study was performed by monitoring fluorescent intensity changes of the $\mathrm{ZnO}$ nanoparticles in a $\mathrm{pH} 1.2$ solution for 8 hours. Separation of Cy5.5 from the Cy5.5-conjugated $\mathrm{ZnO}$ nanoparticles was investigated in saline and $\mathrm{HCl}$ solution $(0.7 \% \mathrm{v} / \mathrm{v}, \mathrm{pH}$ 1.2) for 7 hours. The same volume of Cy5.5-conjugated $\mathrm{ZnO}$ nanoparticles and saline or $\mathrm{HCl}$ solution was poured into vials, and the solution was incubated for 8 hours at $37^{\circ} \mathrm{C}$. Cy5.5conjugated $\mathrm{ZnO}$ nanoparticles were collected by centrifugation at designated time points, and the optical signal intensities of the $\mathrm{ZnO}$ nanoparticles were analyzed using an IVIS spectrum small animal in vivo imaging system (Caliper Lifescience, Hopkinton, MA).

\section{Animal near infrared fluorescent optical imaging study}

Sprague-Dawley rats (5-6 weeks old and weighing 180$200 \mathrm{~g}$ ) were obtained from Orient Bio Inc (Seongnam, Korea). All animal experiments were performed in accordance with the guidelines suggested by the Chonbuk National University Medical School ethics committee. Optical images for Cy5.5-conjugated $\mathrm{ZnO}$ nanoparticles were acquired using the IVIS system. Cy5.5-NHS (1 mg in $0.5 \mathrm{~mL}$ distilled water) or Cy5.5-conjugated $\mathrm{ZnO}$ nanoparticles $(5 \mathrm{mg}$ in $0.5 \mathrm{~mL}$ distilled water) were administrated orally to the rats. In vivo near infrared fluorescent optical imaging was performed at 1 , 2, 3, 5 and 7 hours after administration, using a Cy5.5 filter with the following settings: exposure time (3 s), f/stop (2), binning (8), and field of view (12.8). To investigate absorption into the body from the gastrointestinal tract, blood was collected from the tail vein of each rat 7 hours after ingestion of the nanoparticles, and near infrared fluorescent intensities were estimated using the IVIS system. After acquisition of in vivo optical images, the rats were sacrificed and their major organs, i.e. the heart, lung, liver, spleen, pancreas, kidney, muscle, and bone, were imaged ex vivo. 


\section{Results}

\section{Characterization of Cy5.5-conjugated $\mathrm{ZnO}$ nanoparticles}

In this study, $\mathrm{ZnO}$ nanoparticles were chemically modified and conjugated using Cy5.5, a near infrared fluorescent dye, and traced using an optical imaging system. As shown in Figure 1, the $\mathrm{ZnO}$ nanoparticles were surface-modified with citrate. The amine group was substituted with EBEA on their surfaces to tag an optical dye. The presence of amine groups on the surfaces of the $\mathrm{ZnO}$ nanoparticles was characterized by Fourier transform infrared spectroscopy. Figure 2 shows the infrared spectra of $\mathrm{ZnO}$ nanoparticles and nanoscaled amino-citrate $\mathrm{ZnO}$ nanoparticles. In the infrared spectra of the amino-citrate $\mathrm{ZnO}$ nanoparticles, specific peaks of EBEA were observed at 2918, 2842, 1593, and $1410 \mathrm{~cm}^{-1}$, assigned to amide (I), amide (II), C-O-C, and $\mathrm{CH}_{2} \mathrm{CH}_{3}$ stretching, respectively. Successful conjugation of Cy5.5 with amino-citrate $\mathrm{ZnO}$ nanoparticles was confirmed using phantom optical imaging. As shown in Figure 3, we found a strong near infrared fluorescent signal from Cy5.5-conjugated $\mathrm{ZnO}$ nanoparticles at low concentrations. We measured the tagging stability of Cy5.5 on $\mathrm{ZnO}$ nanoparticles at $\mathrm{pH} 1.2$, which is similar to gastric $\mathrm{pH}$ (Figure 4). Cy5.5 conjugation of the nanoscaled $\mathrm{ZnO}$ nanoparticles was stable for 3 hours (Figure 4A). Although the fluorescent intensity of the nanoparticles decreased after that time, the intensity of Cy5.5 conjugation was maintained above $68 \%$ of its initial intensity until 8 hours. On the other hand, in the case of the submicronscaled $\mathrm{ZnO}$ nanoparticles, the fluorescent intensity was stable for 8 hours in $\mathrm{pH} 1.2$ solution without separation of Cy5.5 from the $\mathrm{ZnO}$ nanoparticles (Figure 4B).

\section{Optical imaging studies after oral administration}

Figure 5 shows the fluorescent intensity of Cy5.5-NHS and $\mathrm{Cy} 5.5$-conjugated $\mathrm{ZnO}$ nanoparticles in rat blood for 7 hours after oral administration. The optical signal intensity in rat blood after Cy5.5-NHS was orally administered to the rats showed a significant increase up to 3 hours, but the signal decreased rapidly thereafter (Figure 5A). In contrast, when Cy5.5-conjugated $\mathrm{ZnO}$ nanoparticles were orally administered, the signal in blood showed different profiles when compared with Cy5.5-NHS (Figure 5B and 5C). Cy5.5-conjugated nanoscaled $\mathrm{ZnO}$ nanoparticles showed the strongest optical signal intensity at 4 hours after oral administration, and the signal intensity decreased after that time. The signal intensity in rat blood increased gradually for 7 hours after administration of Cy5.5-conjugated submicronscaled $\mathrm{ZnO}$ nanoparticles. The signal intensity profiles after oral administration of Cy5.5-NHS or Cy5.5-conjugated ZnO nanoparticles demonstrate that the $\mathrm{ZnO}$ nanoparticles were absorbed into the blood after oral administration and that the Cy5.5 conjugated onto the $\mathrm{ZnO}$ nanoparticles remained stable in the gastrointestinal tract.

Figure 6 shows whole rat body optical fluorescence images after oral administration of Cy5.5-NHS or Cy5.5conjugated $\mathrm{ZnO}$ nanoparticles. Movement of Cy5.5-NHS into the intestine from the stomach was more rapid than for the $\mathrm{ZnO}$ nanoparticles (Figure 6A). In particular, the optical signal intensity in the body was observed 3 hours after administration of Cy5.5-NHS. When compared with nanoscaled

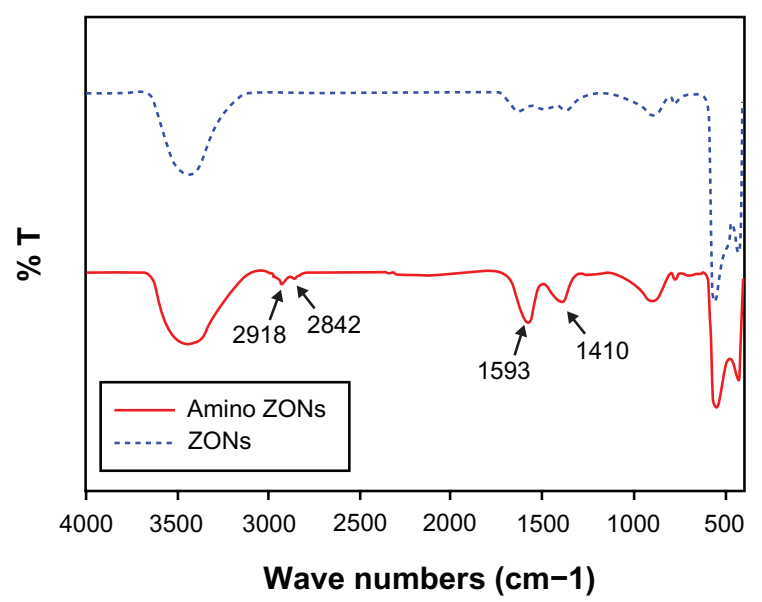

Figure 2 Fourier transform infrared spectra of nonmodified zinc oxide nanoparticles (dotted blue line) and amino-zinc oxide nanoparticles (solid red line).

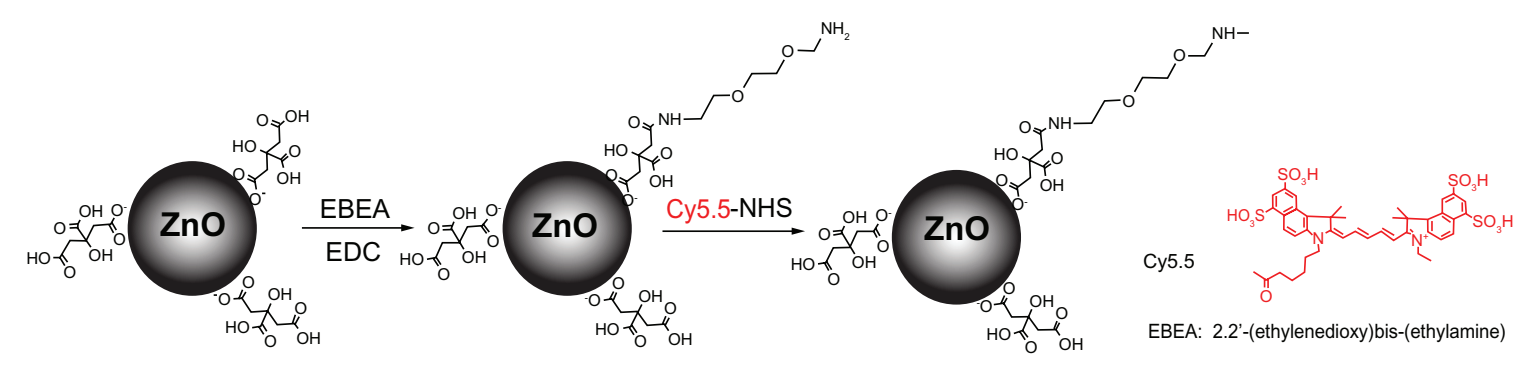

Figure I Schematic illustration of synthesis of Cy5.5-conjugated zinc oxide nanoparticles. 
and submicron-scaled $\mathrm{ZnO}$ nanoparticles, Cy5.5-conjugated nanoscaled $\mathrm{ZnO}$ nanoparticles moved more rapidly into the gastrointestinal tract from the stomach. Cy5.5-conjugated submicron-scaled $\mathrm{ZnO}$ nanoparticles remained for longer in the stomach than did the Cy5.5-conjugated nanoscaled $\mathrm{ZnO}$ nanoparticles. There were significant differences in movement, accumulation, and absorption after oral administration between Cy5.5-NHS and the nanoscaled and submicronscaled $\mathrm{ZnO}$ nanoparticles.

To confirm the biodistribution of Cy5.5-NHS and Cy5.5conjugated $\mathrm{ZnO}$ nanoparticles, we performed ex vivo optical imaging after in vivo imaging 7 hours after administration (Figure 7). As shown in Figure 7A and 8A, Cy5.5-NHS was mostly distributed to the lung, liver, and kidney, and signal intensities were observed in other organs, including the heart, spleen, and pancreas. However, in the case of the Cy5.5-conjugated $\mathrm{ZnO}$ nanoparticles, signal intensities were observed in the liver and kidney (Figures 7B, 7C, 8B, and $8 \mathrm{C})$.

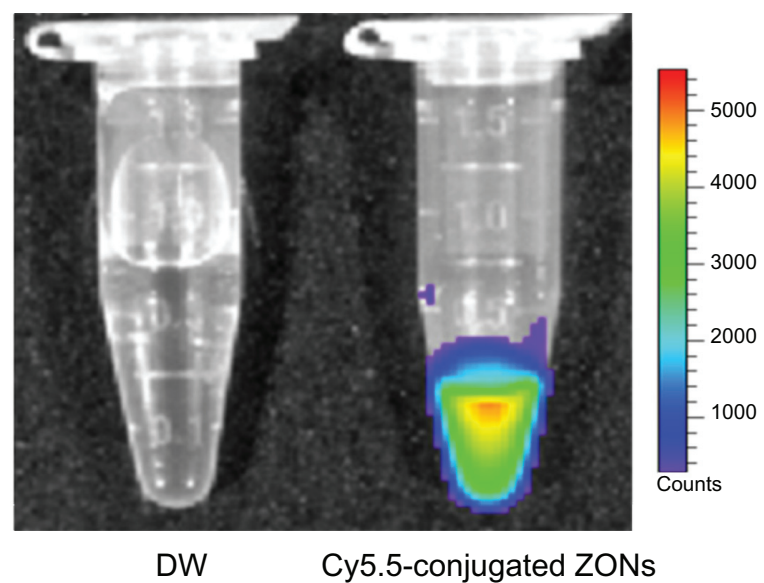

Figure 3 Optical images of distilled water (left) and Cy5.5-conjugated zinc oxide nanoparticles (right)

\section{A}

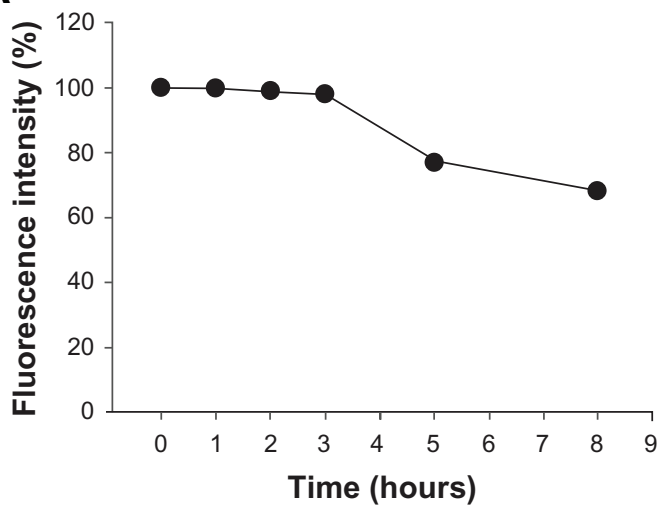

\section{Discussion}

The rapid growth of nanotechnology could have unpredictable consequences for the human body. Nanoparticles entering the body as environmental pollutants can be distributed to various organs, including the lung, liver, kidney, spleen, intestine, and stomach. ${ }^{11-13}$ Orally administered nanoparticles are absorbed into the circulation via the site of entry. When nanoparticles are orally administered, they are immobilized within the submucosal layer of the stomach and Peyer's patches, and then transported quickly into the serosal layer and systemic circulation. ${ }^{14}$ Nanoparticles translocated into the systemic circulation become primarily associated with alveolar macrophages in the lung and Kupffer cells in the liver. ${ }^{11}$ Exposure to $\mathrm{ZnO}$ nanoparticles via the cutaneous, inhalational, and oral routes is now increasing, and studies concerning their toxicity, absorption, and distribution are being conducted. Sharma et $\mathrm{al}^{8,9}$ reported that oral exposure to $\mathrm{ZnO}$ nanoparticles in mice leads to accumulation of nanoparticles in the liver, causing oxidative stress-mediated DNA damage and apoptosis. However, the majority of the studies have been performed in vitro, and the absorption and distribution in the organs after exposure has been evaluated invasively by quantifying existing objective elements in the organs using inductively coupled plasma/mass spectrometry or atomic absorption spectrometry. ${ }^{15,16}$ In this study, we used noninvasive imaging to investigate the distribution and absorption of nanoparticles in the body after exposure. When the nanoparticles are labeled with a radioisotope or a fluorescent dye, their behavior in the body can be monitored and traced using molecular imaging techniques. The information obtained about nanoparticles from such imaging may be used to understand their pharmacokinetics and toxicity.

In this study, we investigated the absorption and distribution of $\mathrm{ZnO}$ nanoparticles, which have broad applications

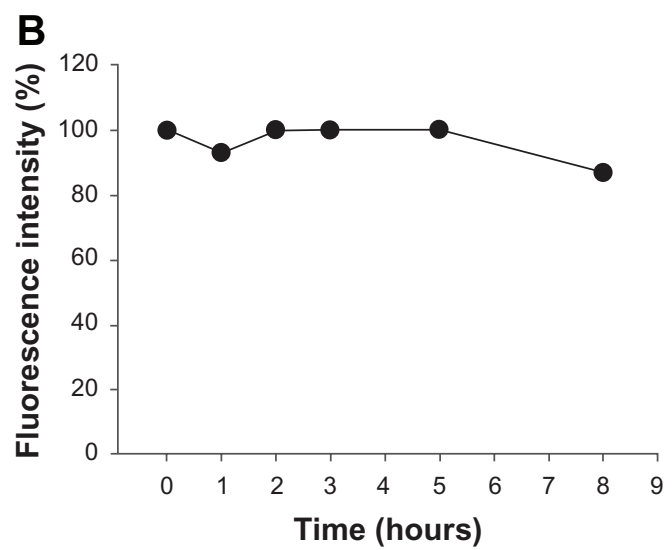

Figure 4 In vitro stability study for Cy5.5 conjugation on (A) nanoscaled and (B) submicron-scaled zinc oxide nanoparticles in $\mathrm{HCl}$ solution (pH I.2). 

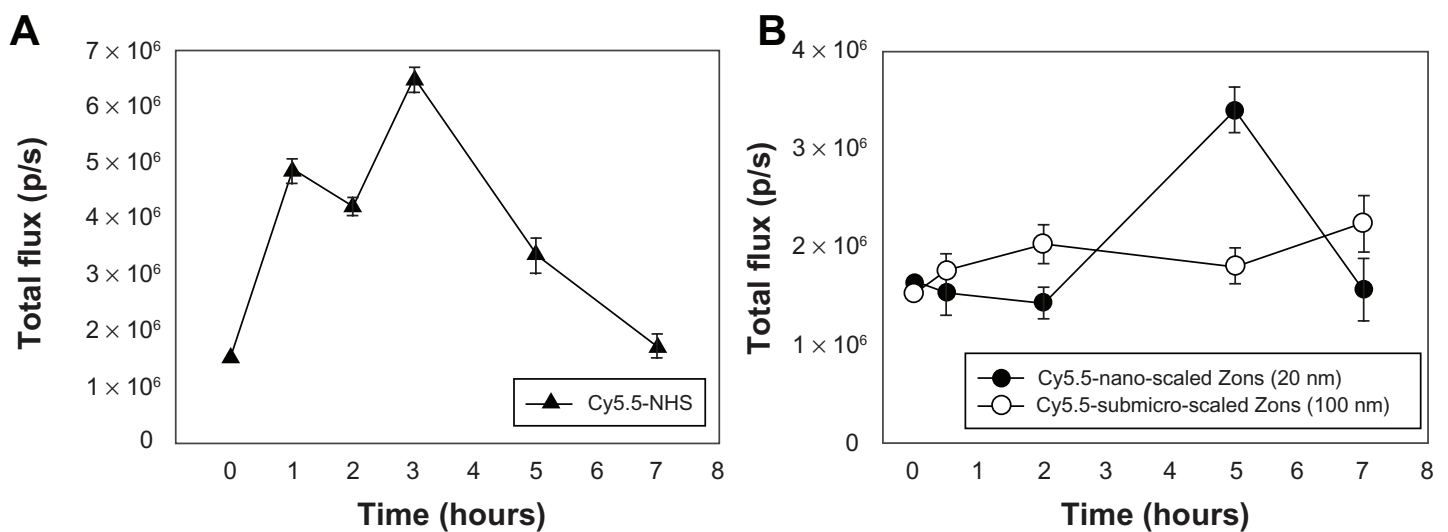

Figure 5 In vivo translocation of (A) Cy5.5-NHS and (B) Cy5.5-conjugated zinc oxide nanoparticles into the blood after oral administration.

A

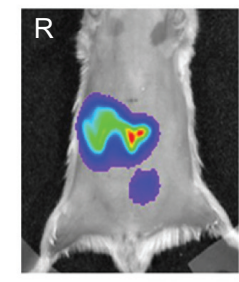

B

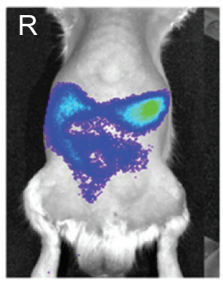

C

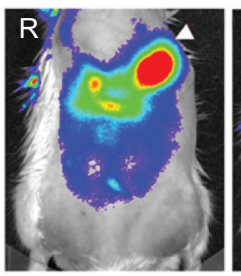

$1 \mathrm{~h}$
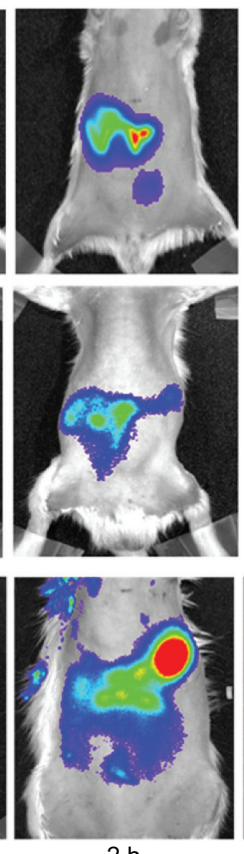

$2 \mathrm{~h}$
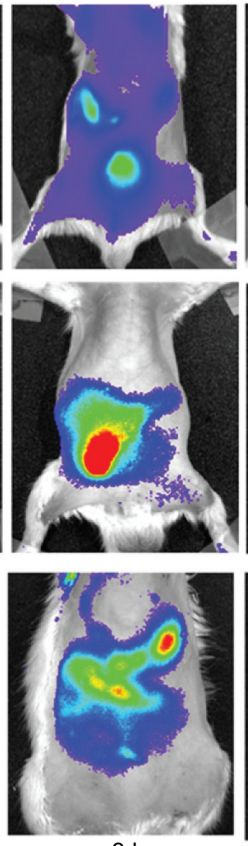

$3 \mathrm{~h}$
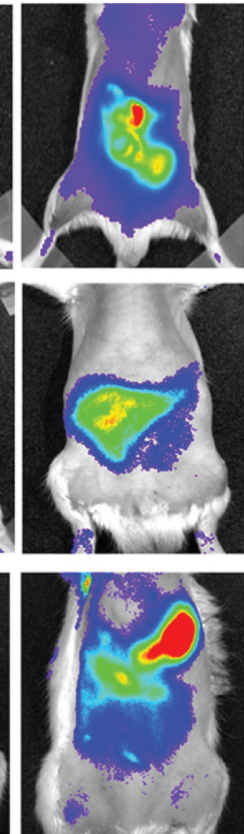

$5 \mathrm{~h}$
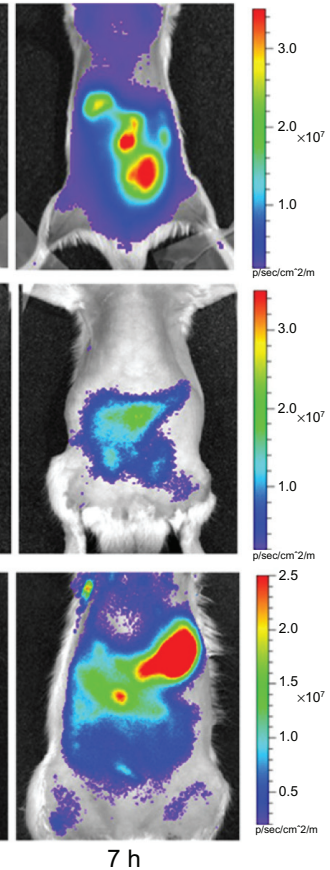

Figure 6 In vivo optical images of rats orally administered with (A) Cy5.5-NHS, (B) Cy5.5-conjugated nanoscaled zinc oxide nanoparticles, and (C) Cy5.5-conjugated submicron-scaled zinc oxide nanoparticles at hours I, 2, 3, 5, and 7 after administration.

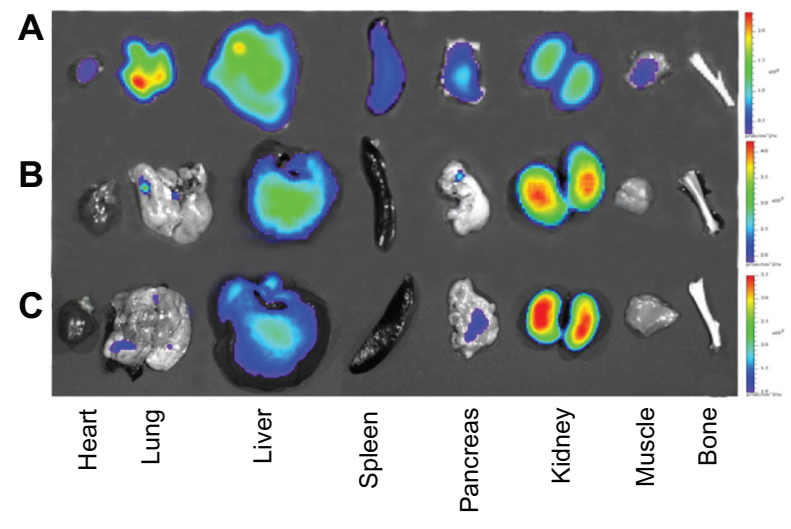

Figure 7 Ex vivo optical images of major rat organs after oral administration with (A) Cy5.5-NHS, (B) Cy5.5-conjugated nanoscaled zinc oxide nanoparticles, and (C) Cy5.5-conjugated submicron-scaled zinc oxide nanoparticles at 7 hours after administration. in diagnostics, drug delivery, foods, paints, and cosmetics, after oral exposure using an optical imaging technique. As shown in Figure 1, the $\mathrm{ZnO}$ nanoparticles were tagged with Cy5.5, a traceable molecule, by chemical modification. The main drawback of tagging with traceable molecules is potential detachment from the nanoparticles via spontaneous or enzyme-catalyzed reactions in vivo. ${ }^{17-20}$ The dissociation of Cy5.5 from nanoscaled $\mathrm{ZnO}$ nanoparticles after 3 hours is inconsequential because the gastric emptying rate is faster than the time needed to dissociate Cy5.5. ${ }^{21}$

Orally administered $\mathrm{ZnO}$ nanoparticles are absorbed into the blood, distributed into various organs, and cleared from the body via the urine. To monitor the absorption of $\mathrm{ZnO}$ nanoparticles after oral administration, we mea- 
sured their signal intensity in blood using the IVIS system (Figure 5). Cy5.5-NHS showed a high signal intensity in the blood 3 hours after oral administration, whereas Cy5.5conjugated $\mathrm{ZnO}$ nanoparticles showed different signal intensity profiles. The different pattern of absorption into the bloodstream shown by Cy5.5-conjugated nanoscaled and submicron-scaled $\mathrm{ZnO}$ nanoparticles can be explained from their different retention times in the stomach. Figure 6 shows whole rat body fluorescent images after oral administration of Cy5.5-NHS or Cy5.5-conjugated $\mathrm{ZnO}$ nanoparticles. The fluorescent signal in the rat body after Cy5.5-NHS administration indicates that it was absorbed and circulated in the blood after oral administration. Cy5.5NHS accumulated in various organs, including the lung and liver (Figure $6 \mathrm{~A}$ ). In the case of rats orally administered Cy5.5-conjugated $\mathrm{ZnO}$ nanoparticles, the signal intensity was mainly observed in the gastrointestinal tract (Figure 6B and $6 \mathrm{C}$ ). These results indicate that a considerable portion of Cy5.5-conjugated $\mathrm{ZnO}$ nanoparticles reached the rat gastrointestinal tract. When Cy5.5-conjugated nanoscaled $\mathrm{ZnO}$ nanoparticles reached the rat stomach, its gastric clearance was faster than that of Cy5.5-conjugated submicron-scaled $\mathrm{ZnO}$ nanoparticles. Gastric emptying rates and absorption are largely dependent on the size and surface properties of particles. ${ }^{22,23}$ In general, smaller particles show faster gastric emptying and absorption into the blood than do larger particles. To identify relatively weak fluorescent signals in organs other than the gastrointestinal tract, we performed ex vivo optical imaging studies (Figure 7). In the case of Cy5.5-NHS, the signals in various organs, including the lung and liver, were observed using in vivo optical images. Ex vivo images from mice administered Cy5.5-conjugated $\mathrm{ZnO}$ nanoparticles demonstrated that $\mathrm{ZnO}$ nanoparticles were absorbed into the blood and distributed mainly in the liver and kidneys. As shown in Figure 8, Cy5.5-conjugated $\mathrm{ZnO}$ nanoparticles showed a different pattern of distribution to the organs after oral administration using Cy5.5-NHS. These results indicate that the optical signal when Cy5.5-conjugated $\mathrm{ZnO}$ nanoparticles were ingested did not proceed from Cy5.5 dissociated from the $\mathrm{ZnO}$ nanoparticles during absorption and distribution after oral administration. When $\mathrm{ZnO}$ nanoparticles were orally administered, and while bowel movements were present, some of them were absorbed into the blood and then mainly distributed in the liver and finally cleared from the body via the urine.

\section{Conclusion}

In this study, we showed that movement of $\mathrm{ZnO}$ nanoparticles after oral exposure could be monitored by optical imaging. Cy5.5 conjugation onto the nanoparticles was sufficiently stable to evaluate the movements of $\mathrm{ZnO}$ nanoparticles in the body after oral administration. Most of the Cy5.5conjugated nanoparticles were excreted via the feces, with some absorbed into the blood and distributed to the organs. Cy5.5-conjugated nanoscaled $\mathrm{ZnO}$ nanoparticles showed faster passage from the stomach into the gastrointestinal tract and absorption into the blood compared with submicron-scaled $\mathrm{ZnO}$ nanoparticles. In view of these results, it is suggested that optical imaging studies may provide the information necessary to understand and investigate the toxicity of $\mathrm{ZnO}$ nanoparticles.

\section{Acknowledgments}

This study was supported by grants from the Korea Food and Drug Administration (10182 KFDA991) and the National R and D Program for Cancer Control, Ministry of Health, Welfare and Family Affairs, Republic of Korea (0620220). This study was also supported by the National Research Foundation of Korea Grant funded by the Korean Government (Ministry of Education, Science and Technology) [NRF-2010-359-D00004].
A

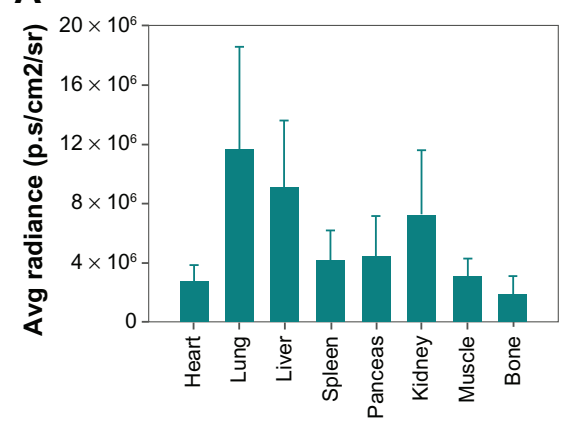

B

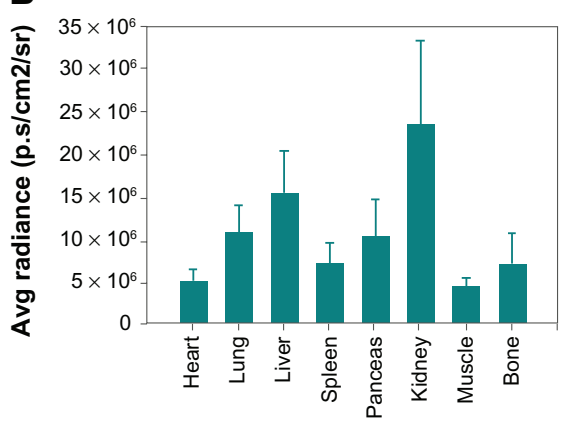

C

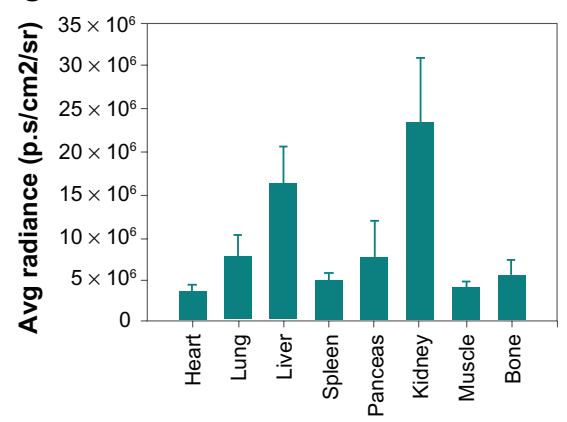

Figure 8 Quantification of ex vivo images after oral administration of (A) Cy5.5-NHS, (B) Cy5.5-conjugated nanoscaled zinc oxide nanoparticles, and (C) Cy5.5-conjugated submicron-scaled zinc oxide nanoparticles at 7 hours after administration. 


\section{Disclosure}

The authors report no conflicts of interest in this work.

\section{References}

1. Jokerst JV, Gambhir SS. Molecular imaging with theranostic nanoparticles. Acc Chem Res. 2011;44:1050-1060.

2. Ray P. Multimodality molecular imaging of disease progression in living subjects. J Biosci. 2011;36:499-504.

3. Luo S, Zhang E, Su Y, Cheng T, Shi C. A review of NIR dyes in cancer targeting and imaging. Biomaterials. 2011;32:7127-7138.

4. Nerl HC, Cheng C, Goode AE, et al. Imaging methods for determining uptake and toxicity of carbon nanotubes in vitro and in vivo. Nanomedicine (Lond). 2011;6:6849-6865.

5. Rasmussen JW, Martinez E, Louka P, Wingett DG. Zinc oxide nanoparticles for selective destruction of tumor cells and potential for drug delivery applications. Expert Opin Drug Deliv. 2010;7:1063-1077.

6. Hackenberg S, Zimmermann FZ, Scherzed A, et al. Repetitive exposure to zinc oxide nanoparticles induces DNA damage in human nasal mucosa mini organ cultures. Environ Mol Mutagen. 2011;52:582-589.

7. Teow Y, Asharani PV, Hande MP, Valiyaveettil S. Health impact and safety of engineered nanomaterials. Chem Commun. 2011;47:7025-7038.

8. Sharma V, Singh P, Pandey AK, Dhawan A. Induction of oxidative stress, DNA damage and apoptosis in mouse liver after sub-acute oral exposure to zinc oxide nanoparticles. Mutat Res. 2012;745:84-91.

9. Sharma V, Anderson D, Dhawan A. Zinc oxide nanoparticles induce oxidative DNA damage and ROS-triggered mitochondria mediated apoptosis in human liver cells (HepG2). Apoptosis. March 7, 2012. [Epub ahead of print].

10. Chen JK, Shih MH, Peir JJ, et al. The use of radioactive zinc oxide nanoparticles in determination of their tissue concentrations following intravenous administration in mice. Analyst. 2010;135:1742-1746.

11. Li CH, Shen CC, Cheng YW, et al. Organ biodistribution, clearance, and genotoxicity of orally administered zinc oxide nanoparticles in mice. Nanotoxicology. September 27, 2011. [Epub ahead of print].

12. Maneewattanapinyo P, Banlunara W, Thammacharoen C, Ekgasit S, Kaewamatawong T. An evaluation of acute toxicity of colloidal silver nanoparticles. J Vet Med Sci. 2011;73:1417-1423.
13. Loeschner K, Hadrup N, Qvortrup K, et al. Distribution of silver in rats following 28 days of repeated oral exposure to silver nanoparticles or silver acetate. Part Fibre Toxicol. 2011;8:18.

14. Eldridge JH, Hammond CJ, Meulbroek JA, Staas JK, Gilley RM, Tice TR. Controlled vaccine release in the gut-associated lymphoid tissues. I. Orally administered biodegradable microspheres target the Peyer's patches. J Control Release. 1990;11:205-214.

15. Crayton SH, Elias DR, Al Zaki A, Cheng Z, Tsourkas A. ICP-MS analysis of lanthanide-doped nanoparticles as a non-radiative, multiplex approach to quantify biodistribution and blood clearance. Biomaterials. 2012:33:1509-1519.

16. Pasupuleti S, Alapati S, Ganapathy S, Anumolu G, Pully NR, Prakhya BM. Toxicity of zinc oxide nanoparticles through oral route. Toxicol Ind Health. October 27, 2011. [Epub ahead of print].

17. Sidhu P, Garg ML, Dhawan DK. Effect of zinc on biological half-lives of ${ }^{65} \mathrm{Zn}$ in whole body and liver and on distribution of ${ }^{65} \mathrm{Zn}$ in different organs of rats following nickel toxicity. Biol Trace Elem Res. 2004; 102:173-188.

18. Hallmans G, Lidén S. Penetration of ${ }^{65} \mathrm{Zn}$ through the skin of rats. Acta Derm Venereol. 1979;59:105-112.

19. Zvyagin AV, Zhao X, Gierden A, Sanchez W, Ross JA, Roberts MS Imaging of zinc oxide nanoparticle penetration in human skin in vitro and in vivo. J Biomed Opt. 2008;13:064031.

20. Areses P, Agüeros MT, Quincoces G, et al. Molecular imaging techniques to study the biodistribution of orally administered ${ }^{99 \mathrm{~m}} \mathrm{Tc}$ labelled naive and ligand-tagged nanoparticles. Mol Imaging Biol. 2011;13:1215-1223.

21. Newton JM. Gastric emptying of multi-particulate dosage forms. Int J Pharm. 2010;395:2-8.

22. Xia D, Cui F, Piao H, et al. Effect of crystal size on the in vitro dissolution and oral absorption of nitrendipine in rats. Pharm Res. 2010;27:1965-1976.

23. Awaad A, Nakamura M, Ishimura K. Imaging of size-dependent uptake and identification of novel pathways in mouse Peyer's patches using fluorescent organosilica particles. Nanomedicine. September 1, 2011. [Epub ahead of print].
International Journal of Nanomedicine

\section{Publish your work in this journal}

The International Journal of Nanomedicine is an international, peerreviewed journal focusing on the application of nanotechnology in diagnostics, therapeutics, and drug delivery systems throughou the biomedical field. This journal is indexed on PubMed Central,

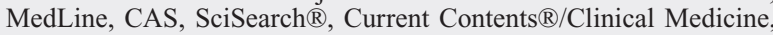

\section{Dovepress}

Journal Citation Reports/Science Edition, EMBase, Scopus and the Elsevier Bibliographic databases. The manuscript management system is completely online and includes a very quick and fair peer-review system, which is all easy to use. Visit http://www.dovepress.com/ testimonials.php to read real quotes from published authors. 